\title{
Contrast variation SANS measurement of shell monomer density profiles of smart core-shell microgels
}

\author{
Marian Cors ${ }^{1,2}$, Lars Wiehemeier ${ }^{1}$, Oliver Wrede ${ }^{1}$, Artem Feoktystov $^{3}$, Fabrice Cousin $^{4}$, Thomas \\ Hellweg ${ }^{1 *}$, Julian Oberdisse ${ }^{2 *}$ \\ ${ }^{1}$ Department of Physical and Biophysical Chemistry, Bielefeld University, Universitätsstr. 25, 33615 \\ Bielefeld, Germany \\ ${ }^{2}$ Laboratoire Charles Coulomb (L2C), University of Montpellier, CNRS, 34095 Montpellier, France. \\ ${ }^{3}$ Forschungszentrum Jülich GmbH, Jülich Centre for Neutron Science JCNS at Heinz Maier-Leibnitz. \\ Zentrum MLZ, 85748 Garching, Germany. \\ ${ }^{4}$ Laboratoire Léon Brillouin, UMR 12 CEA/CNRS, CEA Saclay, 91191 Gif Sur Yvette, France \\ *Authors for correspondence : thomas.hellweg@uni-bielefeld.de, julian.oberdisse@umontpellier.fr
}

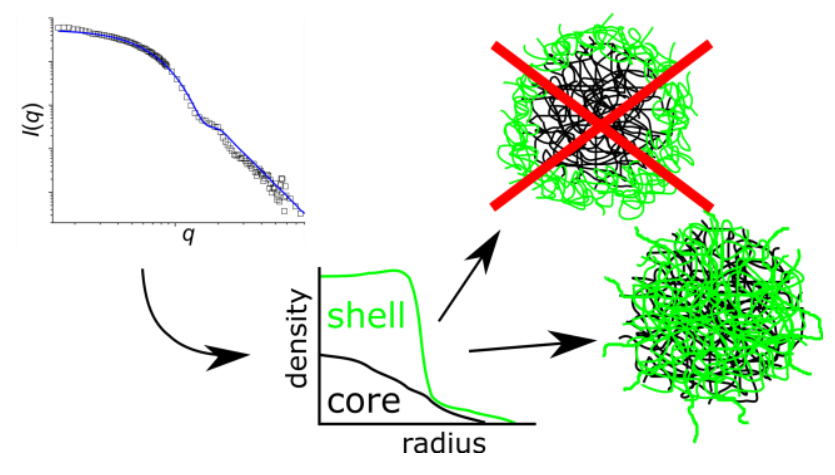

\begin{abstract}
The radial density profile of deuterated poly( $N, n$-propyl acrylamide) shell monomers within core-shell microgels has been studied by small angle neutron scattering in order to shed light on the origin of their linear thermally-induced swelling. The poly( $N$-isopropyl methacrylamide) core monomers have been contrast-matched by the $\mathrm{H}_{2} \mathrm{O} / \mathrm{D}_{2} \mathrm{O}$ solvent mixture, and the intensity thus provides a direct measurement of the spatial distribution of the shell monomers. Straightforward modelling shows that their structure does not correspond to the expected picture of a well-defined external shell. A multi-shell model solved by a reverse Monte Carlo approach is then applied to extract the monomer density as a function of temperature and of the core crosslinking. It is found that most shell monomers fill the core at high temperatures approaching synthesis conditions of collapsed particles, forming only a dilute corona. As the core monomers tend to swell at lower temperatures, a skeleton of insoluble shell monomers hinders swelling, inducing the progressive linear thermoresponse.
\end{abstract}




\section{Introduction}

Acrylamide-based microgel particles reply to external stimuli, in particular temperature, by size changes via swelling by the solvent. ${ }^{1-10}$ This behaviour grants them the adjective smart. The response of these materials has great potential in the context of different applications like smart catalyst carriers, ${ }^{11-13}$ drug delivery, ${ }^{14,15}$ sensors, ${ }^{16-18}$ optical devices, ${ }^{19-21}$ responsive surface coatings ${ }^{22}$ and actuators ${ }^{23}$ or in biomedical applications. ${ }^{24}$ Combining two monomers of different transition temperatures in statistical copolymers can be used to tune the swelling transition temperature, ${ }^{25-29}$ whereas the subsequent synthesis of core and then shell ${ }^{27,30-35}$ may lead to a continuous, linear temperature dependent swelling. ${ }^{27,36,37}$ The linearity of the response of these coreshell systems might be beneficial especially for sensors and actuators. An explanation of the restricted swelling of the core in the linear regime has been proposed and termed the "corset effect". Its idea is that the shell surrounding the core does not swell at the same temperature, thereby impeding the extension of the core. Obviously, this can only work if the shell monomers form indeed a well-defined shell around the preformed core.

The structure of such core-shell microgel particles has been measured by small-angle neutron scattering (SANS) and the data were analysed with a variety of core-shell models, ${ }^{38-41}$ where the contrast situation in absence of deuteration did not provide the possibility of discriminating the two monomers. Naturally, it is difficult to conclude then on the existence of well-defined shells, or on possible interpenetration. It is even more complicated to study the evolution of, e.g., interpenetration with synthesis parameters and geometry, or particle size. The existence of core-shell structures has been deduced from the beginning by scattering without isotopic labelling, in combination with swelling curves, ${ }^{38-41}$ or more recently by using fluorophore labelling and high resolution optical microscopy. ${ }^{42,43}$ Core-shell models for small-angle scattering are based on an analytic description of the radial density profile, allowing Fourier transformation for comparison with the measured intensities and adjusting to it by variation of a limited set of parameters: core and shell geometry through radii and densities, as well as fuzziness of the interface(s). ${ }^{40,41,44}$ Typical analytic functions are either Gaussians or piecewise polynomials. Such measurements and analyses confirmed the presence of both core and shell monomers, and seemed to confirm the intuitive assumption that the shell forms just outside the core in the synthesis step. ${ }^{38-41,44}$ In some occasion, form-free modelling has been used to check suitability of such models. ${ }^{45}$ Here "form-free" refers to a general profile defined by a sequence of densities in (thin) concentric shells, each of which can be Fourier-transformed analytically.

Our efforts to understand the striking linear swelling (Figure S1 in the SI) properties of spherical core-shell microgels aim at a detailed and form-free description of their internal microstructure using contrast-variation SANS and an analysis giving access to the density profile of both core and shell monomers. The absence of a priori assumptions on the internal architecture of the density profiles proved to be crucial, as the geometries 
turned out to be quite unexpected. Our approach is based on the combination of several measurements, starting with a pure poly( $N$-isopropyl methacrylamide) (pNIPMAM) core (“core-only"), onto which either deuterated or hydrogenated poly( $N, n$-propyl acrylamide) (pNNPAM) shells are added. It is emphasized that

all cores are identical in the present and previous studies, ${ }^{46}$ i.e. the shell synthesis has been performed with existing cores, allowing for direct comparisons. For the H-D core-shell microgels, two contrast situations are of particular interest: measurements in $12 \mathrm{v} \% / 88 \mathrm{v} \% \mathrm{H}_{2} \mathrm{O} / \mathrm{D}_{2} \mathrm{O}$ have been used to match the deuterated shell monomers and characterize the cores. We have found quite unexpectedly that the cores embedded in core-shell microgels possess the same mass but occupy a bigger volume than the corresponding 'core-only' particles. ${ }^{46}$ They possess thus a lower volume fraction, in contradiction with any compression induced by the (hypothetical) corset effect. This striking observation could only be explained by swelling of the core by the shell monomers. Indeed, by comparing the H-H with the H-D monomer density profiles, the radial density profile of the shell monomers could be deduced indirectly, necessarily based on the assumption that the different syntheses give rigorously identical distributions. It is the purpose of the present article to directly measure the radial density profile of the shell monomers by contrast-matching the hydrogenated core monomers in $77 \mathrm{v} \% / 23 \mathrm{v} \% \mathrm{H}_{2} \mathrm{O} / \mathrm{D}_{2} \mathrm{O}$ solvent mixtures, and to analyse the resulting SANS curves using a form-free multi-shell reverse Monte Carlo analysis. ${ }^{45,47-51}$

\section{Experimental}

Synthesis: The microgel synthesis were performed under nitrogen atmosphere in a continuously stirred three-necked flask. The flask was equipped with a mechanical stirrer a septum for the nitrogen inlet and a reflux condenser. At first the cross-linker $N, N^{\prime}$-methylenebis(acrylamide) (BIS) (99\%, Sigma-Aldrich, St. Louis, USA) was dissolved in water. After the solution was heated to $70{ }^{\circ} \mathrm{C}$ and purged with nitrogen for $1 \mathrm{~h}$, the core monomer $N$-isopropylmethacrylamide (NIPMAM) (97 \%, Sigma-Aldrich, St. Louis, USA) and the surfactant sodium dodecyl sulfate (SDS) (>99 \%, Sigma-Aldrich, St. Louis, USA) were added. Then the reaction was initiated by adding the radical starter ammonium peroxodisulfate (APS) (>98\%, Sigma-Aldrich, St. Louis, USA). After $4 \mathrm{~h}$ the heating oil bath was removed and the solution was stirred overnight at room temperature. The purification was done by five cycles of centrifugation (Avanti J-30, Beckman Coulter GmbH, Krefeld, Germany) at ca. $50000 \mathrm{~g}$ for at least 30 minutes, decantation and redispersion.

For the shell synthesis the monomer D7- $N-n$-propylacrylamide (D7-NNPAM, Chemical structure in Figure 1) was synthesized by a Schotten-Baumann reaction as described by Hirano et al. using acryloyl chloride (97\% Sigma-Aldrich, St. Louis, USA), triethylamine (99\%, Sigma-Aldrich, St. Louis, USA), 
D7-propylamine (99\%, Sigma-Aldrich, St. Louis, USA) and methylene chloride (99 \%, Sigma-Aldrich, St. Louis, USA). ${ }^{52}$ The deuterated monomer structure was verified by NMR measurements $\left(\mathrm{H}^{1}, \mathrm{H}^{2}\right.$ and $\left.\mathrm{C}^{12}\right)$. The shell synthesis was done following the same protocol as for the core described above, with a crosslinker content of $1.9 \mathrm{~mol} \%$ and the exception that the core particles $(0.15 \mathrm{wt} \%)$ were added as seeds at the same time as the cross-linker. Three different core-shell microgel particles with 5, 10 and 15 mol\% crosslinker in the core seeds synthesis were synthesized. Note that secondary nucleation leading to separate microgel particles of core and shell polymer are not observed, as the resulting core-shell microgels do show a specific swelling behaviour, and moreover FTIR-spectroscopy shows a cooperative effect of the two polymers. $^{37}$

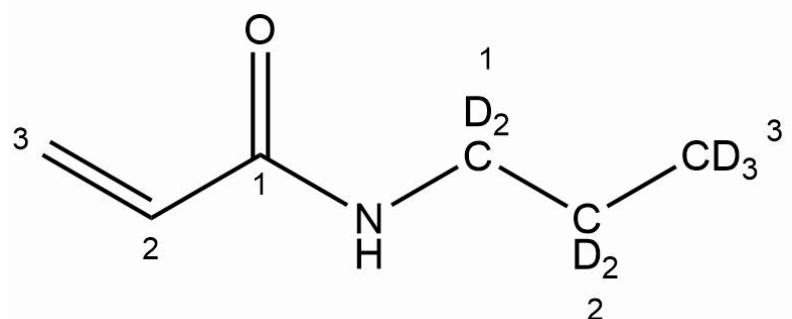

Figure 1: Chemical structure of D7- $N, n$-propyl acrylamide (D7-NNPAM).

SANS Measurements: The SANS measurements were performed at PA20 $0^{53}$ (LLB, Saclay, France) and KWS-1 ${ }^{54}$ (JCNS at MLZ, Garching, Germany). Three different configurations were used to cover the $q$ range of $0.001 \AA^{-1}$ to $0.3 \AA^{-1}$. On the KWS- 1 we used: $1 \mathrm{~m}$ and $\lambda=5 \AA, 8 \mathrm{~m}$ and $5 \AA$, and $20 \mathrm{~m}$ and $12 \AA$. On PA20 we used: $1.5 \mathrm{~m}$ and $4 \AA$, $8 \mathrm{~m}$ and $6 \AA$, and $20 \mathrm{~m}$ and $6 \AA$. The normalization (detector electronic noise, water or Plexiglas, empty cell) of the data was done with QtiKWS (JCNS, Germany) for the data from KWS-1 and with Pasinet software (LLB, Saclay, France) for the data from PA20 to receive scattering data in absolute units.

The scattering length densities (SLD) of the microgel components were determined by contrast variation. The SLD of H-pNIPMAM: $1.0 \cdot 10^{10} \mathrm{~cm}^{-2}$ and the SLD of D7-pNNPAM is $5.54 \cdot 10^{10} \mathrm{~cm}^{-2} \cdot{ }^{46,50}$ The SLD of the solvents were estimated assuming a density of $1.107 \mathrm{~g} / \mathrm{cm}^{3}$ for $\mathrm{D}_{2} \mathrm{O}\left(\mathrm{SLD}=6.38 \cdot 10^{10} \mathrm{~cm}^{-2}\right)$ and of $1.000 \mathrm{~g} / \mathrm{cm}^{3}$ for $\mathrm{H}_{2} \mathrm{O}\left(\mathrm{SLD}=-0.56 \cdot 10^{10} \mathrm{~cm}^{-2}\right)$.

Multi-shell model for radial density profile and reverse Monte Carlo optimization. We described the RMC fit algorithm in previous articles in detail. ${ }^{46,50}$ Its purpose is to determine the radial volume fraction profile $\Phi(r)$ of the monomers probed by the SANS experiments, i.e. of the deuterated monomers, the rest of the available space being occupied by the solvent and the matched hydrogenated core monomers. The 
algorithm has one main assumption, which is that the microgel particles exhibit spherical symmetry, allowing for the representation of the density profile by monomers located in concentric spherical shells at radius $r$, and of thickness $2 \mathrm{~nm}$. Note that the spherical symmetry of the particles has been checked by various real-space images. ${ }^{50,55}$ This allows the RMC algorithm to move randomly packages of monomers between the different shells in order to optimize the agreement between the measured and the model intensity. The intensity is calculated from the monomer density profile including polydispersity $(\sigma=0.2)$, the resolution function, and an ad-hoc addition of a generalized Gaussian coil to describe the chain or surface scattering. ${ }^{56}$ The effect of higher polydispersity is to sharpen the decays of the profiles, and vice versa, which is why it has been determined by direct imaging beforehand..$^{50}$ After each move (MC step) the algorithm calculates the corresponding scattering curve and compares it with the experimental data. Thereby the algorithm follows some rules: (1) The volume fraction of monomers in a shell has to be between 0 and 1 , counting both the known and fixed monomer density profile of the core, and the now measured shell contribution. (2) The algorithm starts with 100 shell monomers per move. This number reduces progressively to 1 . (3) The total monomer density (core + shell) is only allowed to decrease with increasing radius, avoiding empty inner shells. (4) Moves are accepted when the deviation between measured and theoretical curve is reduced or the increase is negligible following a Boltzmann/Metropolis criterion. Simultaneously, minimum noise is sought in the resulting $\Phi(r)$ profile..$^{50}$

\section{Results and discussion}

The synthesis of the core-shell microgel particles in question has been described in detail previously, ${ }^{46,50}$ and the exact protocol of the shell synthesis onto the cores is given in the experimental section. The core mass conservation during the shell synthesis was confirmed by the measured intensity $I(q \rightarrow 0)$ in absolute units $\left(\mathrm{cm}^{-1}\right.$, scattering cross section per unit sample volume) with the same concentration of both samples (core, and core-shell with invisible shell). Besides selective deuteration, a key parameter to be studied is the concentration of core cross-linkers (CCC) $N, N$ '-methylenebis(acrylamide) (BIS). By matching the hydrogenated core monomers in $23 \mathrm{v} \% \mathrm{D}_{2} \mathrm{O}$, only the shell monomers contribute to the coherent intensity. In previous work, we matched the deuterated shell and investigated the hydrogenated core of the same samples under different contrast conditions. ${ }^{46}$ The SANS intensity curve of a pNIPMAM/pNNPAM H-core D7-shell particle with a CCC of $10 \mathrm{~mol} \%$ at $55{ }^{\circ} \mathrm{C}$ in Figure 2a (black squares) illustrates the general aspects of the microgel architecture. The solvent $\left(23 \mathrm{v} \% \quad \mathrm{D}_{2} \mathrm{O}\right)$ matching exactly the core monomers $\left(\rho=1.0 \cdot 10^{10} \mathrm{~cm}^{-2}\right)^{50}$, the entire coherent intensity curve is related to the number and spatial distribution of the shell monomers within the core-shell microgel particles. Main features of the scattered intensity are that 
it displays an almost perfect low- $q$ plateau - there is no peak and only a moderate low- $q$ increase -, indicating only very little interactions between the particles. The Guinier domain can be described by a classical radius of gyration $\left(R_{\mathrm{g}}\right)$ :

$$
I(q)=I_{0} \exp \left(-q^{2} R_{\mathrm{g}}^{2} / 3\right)
$$

The low- $q$ limit $\mathrm{I}(q \rightarrow 0)$ is represented by the prefactor $I_{0}$ allowing the determination of the number of D7-pNNPAM shell monomers in this sample, which is then kept constant at all temperatures. We found monomer numbers of $2.4 \times 10^{5}$, as compared to $1.1 \times 10^{5}$ for the core. The $R_{\mathrm{g}}$ resulting from the fit in Figure $2 \mathrm{a}$ is $28 \mathrm{~nm}$. For hollow shell geometries, $R_{\mathrm{g}}$ is expected to lie within the shell (Fig. S2). Comparison with the radius of gyration of the cores at the same temperature, ${ }^{46} R_{\mathrm{g}}{ }^{\text {core }}=27 \mathrm{~nm}$, which is known to lie inside homogeneous spheres $\left(R^{\text {core }}=\sqrt{5 / 3} R_{\mathrm{g}}^{\text {core }}=35 \mathrm{~nm}\right)$, immediately provides evidence for a non-classical structure: the shell seems to be smaller than the core. Elucidating this striking observation is the main objective of the present article. At intermediate $q$, a damped oscillation characterizing the particle size is found, followed by a high- $q$ power law describing the polymer chain and surface scattering.

A priori, different radial density profiles of shell monomers can be imagined, and before turning to our form-free reverse Monte Carlo analysis, we show that intuitively expected models of the shell density profiles (see SI for details) are incompatible with the observed intensity. In all cases, we have taken into account the known density profile of the core monomers, ${ }^{46}$ thus limiting the space to accommodate further shell monomers. All tentative shell profiles are shown in Figure $2 b$, and the corresponding intensity fits in Figure 2a, respectively.
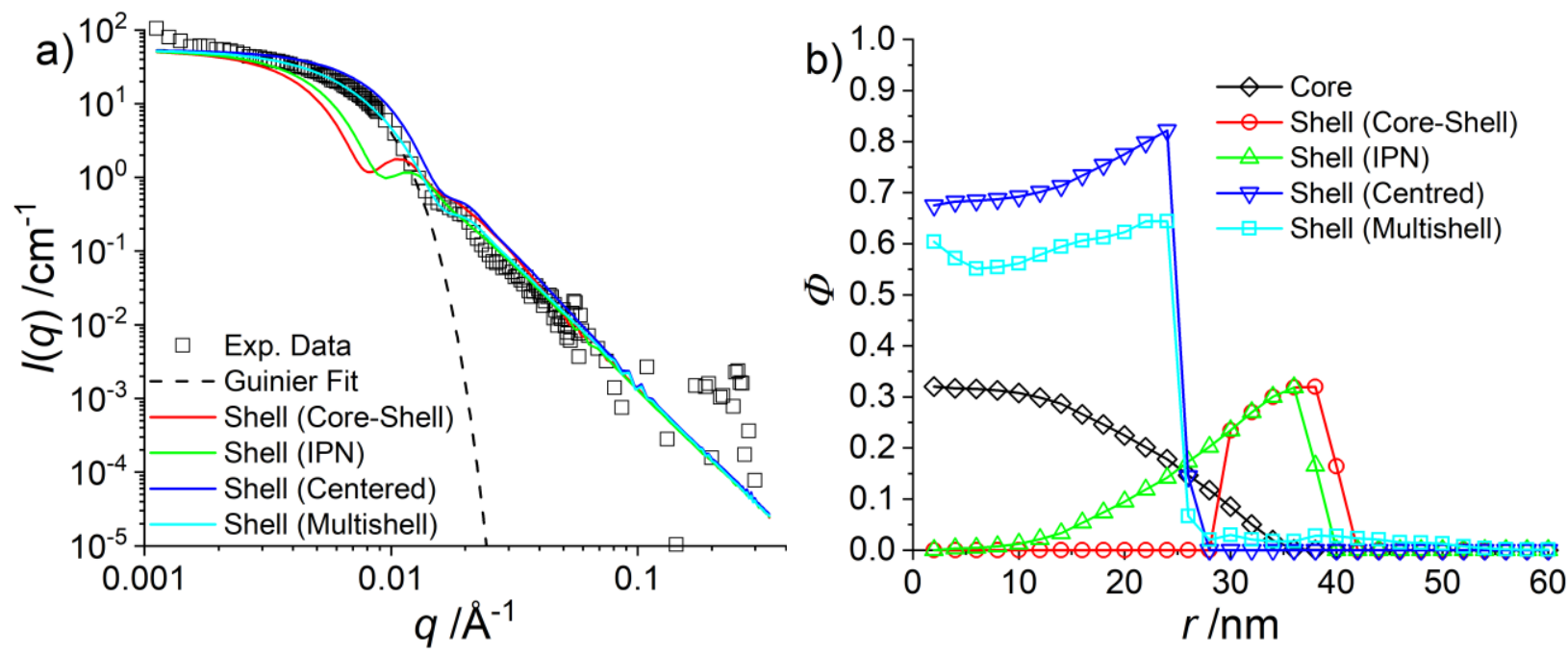

Figure 2: a) Intensity curve of a D7-pNNPAM-shell with a contrast matched H-pNIPMAM-core (black squares) at $55{ }^{\circ} \mathrm{C}$ from a SANS experiment. The CCC is $10 \mathrm{~mol} \%$. The Guinier approximation is shown as dotted line $\left(R_{\mathrm{g}}=28 \mathrm{~nm}\right)$. The coloured lines represent the calculated intensity curves of the different simulated shell density 
profiles in b). b) The measured density profile of the H-pNIPMAM core (black squares, in presence of contrastmatched shell-chains, data adapted from Cors et al ${ }^{46}$ and different density profiles tested for the shell monomers.

The first obvious architecture is a well separated core-shell system with a very small degree of interpenetration. The shell profile (named "Shell (Core-Shell)", red circles in Figure 2b) was tentatively located just outside the known core profile (black squares), with a maximum density identical to the one of the core, thus reaching a thickness of ca. $15 \mathrm{~nm}$. The corresponding calculated scattering curve in Figure $2 \mathrm{a}$ is seen to completely miss the Guinier regime, meaning that the simulated shell geometry is larger than the experimentally measured one. Increasing the interpenetration into the core produced smaller shells, named "Shell (IPN)" in Figure 2b, but the corresponding intensity (green line in Figure 2a) still fails to describe the experimental data because of the too big size. On the other extreme, the smallest possible microgel model is obtained by filling the remaining space with shell monomers, starting from the centre, setting the total volume fraction to $\Phi=1$. The shell density then increases as the experimental core monomer density decreases. It results that all shell monomers can be accommodated in the core as shown in Figure $2 \mathrm{~b}$, and we have termed this model "Shell (Centred)". The corresponding intensity prediction now overestimates the intensity in the intermediate $q$-range, meaning that the particle is too small. However, this prediction is much closer to the experimental values, and it suggests that the interpenetration of the core and the shell is strong.

We then used our RMC fitting algorithm to find a density profile that describes the experimental data correctly. The outcome of the multi-shell model is a density profile ("Shell (Multishell)", cyan in Figure 2b) that is very similar to the "Centred" model. It has a density of ca. 0.6 in the centre with a sharp decrease at a radius of $24 \mathrm{~nm}$, and its scattering curve in Figure 2a describes very well the experimental SANS data. Note that the algorithm had no other restriction than already reported (see RMC details in SI). ${ }^{46,50}$ It can be concluded that the NNPAM-shell monomers strongly interpenetrate the NIPMAM-core, which is the key result of this article. If this were not the case, the resulting particles would be too big, and the Guinier regime could not be described correctly.

Probably the most interesting property of smart acrylamide-based microgels is their swelling behaviour with temperature. In particular, pNIPMAM/pNNPAM core-shell microgel particles show a peculiar linear swelling with $T$ between the two transition temperatures, $T_{\text {NIPMAM }}=45^{\circ} \mathrm{C},{ }^{28,41,57-61}$ and $T_{\text {NNPAM }}=23{ }^{\circ} \mathrm{C} .{ }^{28,58}$ We have therefore studied the evolution of the shell density profile, applying the same contrast condition highlighting the shell, and analysing our data using the multi-shell reverse Monte Carlo approach. In Figure 3a the scattering curves of SANS measurements of the D7-pNNPAM shell with the matched H-pNIPMAM core are shown for five different temperatures $\left(15,30,35,40,55^{\circ} \mathrm{C}\right.$, black symbols). The green lines are the best-fitting scattering curves calculated with the multi-shell RMC model. The agreement with the data is satisfying: the intensity level, the Guinier domain, the oscillation and the high- $q$ intensity are described 
correctly. The latter correspond to the scattering of either the chains in the swollen state, which is described by a low fractal chain dimension, resp. power law exponent, or of the surface of the collapsed particles at high temperature, with Porod-like decays. The exponents are reported in Tables S1 and S2 in the SI. Figure $3 \mathrm{~b}$ shows the corresponding shell density profiles.
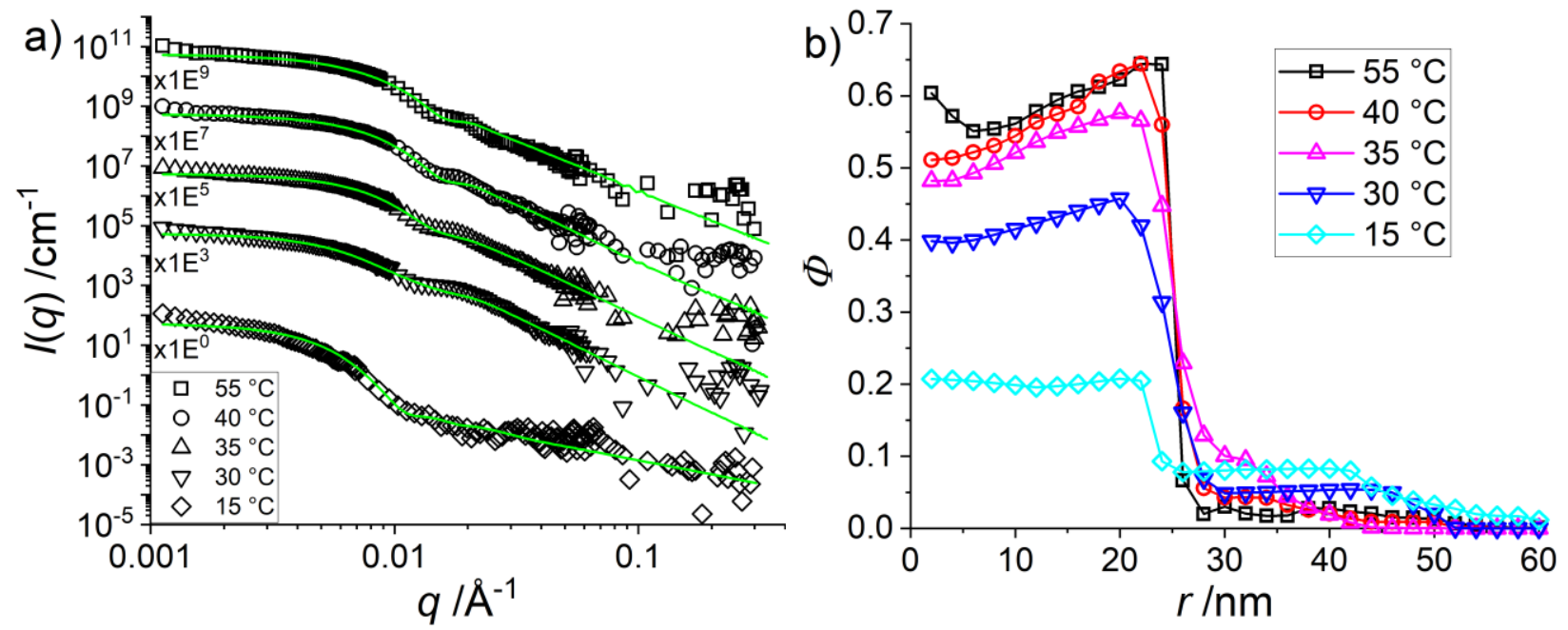

Figure 3: H-pNIPMAM core with D7-pNNPAM shell with a CCC of 10 mol\% (a): I(q) vs. q of SANS measurements with a $\mathrm{H}_{2} \mathrm{O} / \mathrm{D}_{2} \mathrm{O}$ that matches the core. (b): Calculated monomer density profile of the pNNPAM shell from the SANS data in a).

The density profiles of the D7-pNNPAM shell monomers in Figure 3b show a high volume fraction in the particle centre for all temperatures, up to ca. $70 \%$ at the highest temperature. Whatever the temperature, the profiles show a weak increase or constant density up to a drop located at a radius of ca. $26 \mathrm{~nm}$. Then the density reaches a low value around $10 \%$, and eventually vanishes above ca. $50 \mathrm{~nm}$. This drop in shell monomer density is roughly located at the interface of the fully collapsed "core-only" particle (i.e. before synthesis of the shell). This indicates that before the shell synthesis is started at $70{ }^{\circ} \mathrm{C}$, the shell NNPAM monomers are highly enriched in the collapsed pNIPMAM-core, and the polymerization of the shell takes place predominantly in the core, and to a lesser degree on its surface. Consequently, at $55{ }^{\circ} \mathrm{C}$ the shell monomer profile has an extent of ca. $26 \mathrm{~nm}$ and is located inside the core plotted in Figure 2b. A striking feature of Figure $3 \mathrm{~b}$ is that the density profiles are quite similar for all temperatures from $55^{\circ} \mathrm{C}$ down to $30{ }^{\circ} \mathrm{C}$, i.e. below the transition temperature of the core monomers $\left(45^{\circ} \mathrm{C}\right)$ : when the core tends to swell below its transition temperature of $45^{\circ} \mathrm{C}$, the density of shell monomers in the centre is hardly affected. The structure created by the still insoluble shell monomers thus acts as an internal, rigid skeleton at intermediate temperatures. Moreover, shell monomers also form a plateau of constant density around $r=30-45 \mathrm{~nm}$. Only at the lowest temperature, below the shell transition at ca. $23{ }^{\circ} \mathrm{C}$, the shell monomer density in the centre is 
lowered, and water is taken up, accompanied by a significant increase in the plateau region beyond $25 \mathrm{~nm}$. Finally, the microgel system is in the fully swollen state.

The linear swelling of pNIPMAM-pNNPAM "Core-Shell" particles can be tuned by the degree of cross-linking in the pNIPMAM-core. ${ }^{27,36}$ Hence, we performed SANS measurements on a CCC series at high temperature in order to grasp the initial (post-synthesis) density profiles governing the swelling. Note that the BIS concentration for the shell synthesis was set constant to $1.9 \mathrm{~mol} \%$. The intensity curves are shown in Figure 4a, and the corresponding shell monomer density profiles are plotted in Figure 4b.
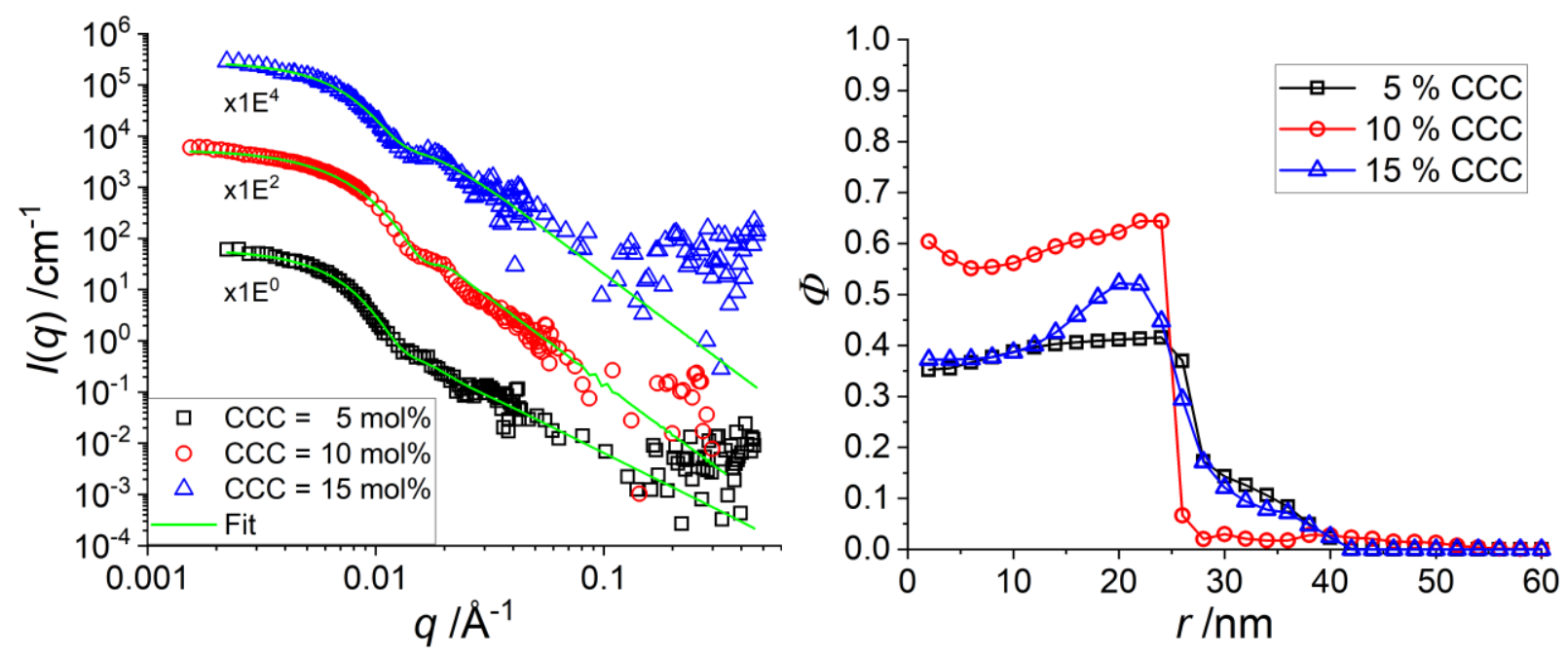

Figure 4: H-pNIPMAM core with D7-pNNPAM shell with different CCC $(5,10,15 \mathrm{~mol} \%)$ at $55{ }^{\circ} \mathrm{C}$. (a): $I(q)$ vs. $q$ of SANS measurements with a $\mathrm{H}_{2} \mathrm{O} / \mathrm{D}_{2} \mathrm{O}$ that matches the core. (b): Calculated monomer density profile of the pNNPAM shell from the SANS data in a).

All three density profiles in Figure $4 \mathrm{~b}$ have a roughly similar shape: a high volume fraction of shell monomers in the centre, followed by a drop around $26 \mathrm{~nm}$, followed by a weak (ca.10\%) shell monomer density up to $40 \mathrm{~nm}$. The reverse Monte-Carlo simulations have been tested for robustness, and consistently predict a higher density in the centre for a $\mathrm{CCC}$ of $10 \mathrm{~mol} \%$, and the presence of a "true" shell for both lower and higher CCC, necessarily due to shell monomer mass conservation. The resulting steeper interface for $10 \mathrm{~mol} \%$ consistently leads to a Porod-like slope at high- $q$, which is not the case for the other crosslinking densities (see Tables S1 and S2 in the SI). Although we do not have any explanation for this non monotonic behaviour, it is thus confirmed by the high- $q$ behaviour independently of our model.

Combining the results of the SANS measurements under different contrast conditions of the same microgel particles - modifying only the $\mathrm{H}_{2} \mathrm{O} / \mathrm{D}_{2} \mathrm{O}$ solvent composition -, we can now compare the volume fraction profiles of the core and the shell monomers. They are presented in Figure 5 for $15^{\circ} \mathrm{C}, 35^{\circ} \mathrm{C}$, and $55^{\circ} \mathrm{C}$. 

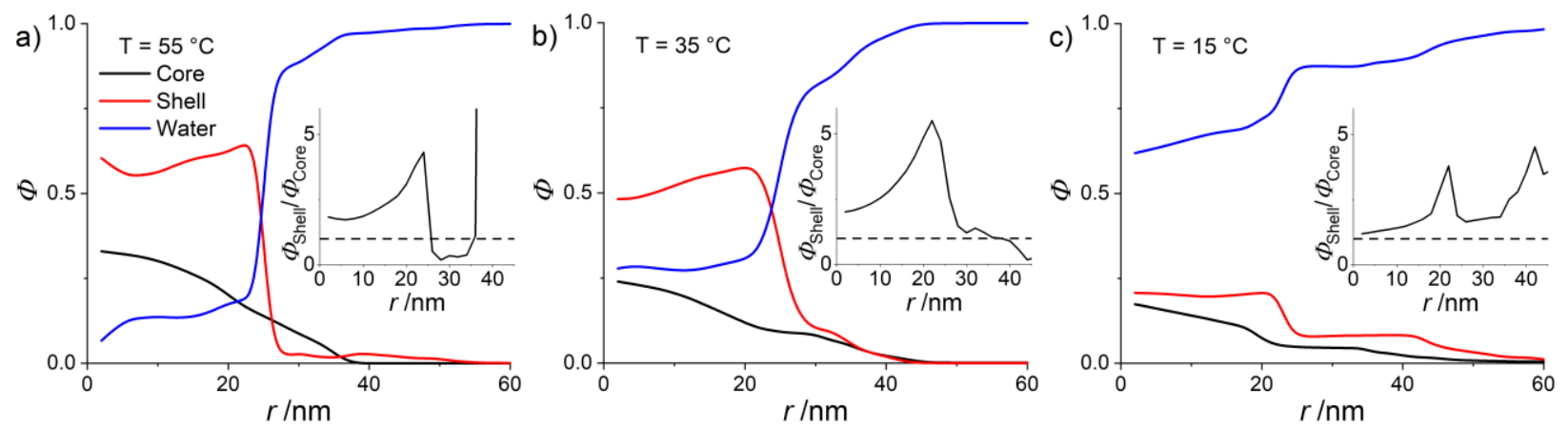

Figure 5: Density profiles of a pNIPMAM core (black line, data adapted from Cors et al., ${ }^{46}$ a D7-pNNPAM "shell“" (red line) and water (blue line) at a) $55{ }^{\circ} \mathrm{C}$, b) $35{ }^{\circ} \mathrm{C}$, and c) $15{ }^{\circ} \mathrm{C}$. The insets represent the ratio of the shell to the core monomer density as a function of radius.

As recognized already when discussing the Guinier domain shown in Figure 2a, many shell monomers are indeed located within the core at all temperatures. This induces a swelling of the core with respect to the size of the "core-only" particle, and changes the steepness of the interface. The resulting volume fraction of water is around $20 \%$ in the core at the highest temperature, and increases up to 60-70\% for the fully swollen microgel. Due to the spherical symmetry, the representation in Figure 5 does not highlight the real monomer quantities at a distance $\mathrm{r}$, and a $r^{2} \Phi$ plot is shown in the SI for comparison (Figure S3). It then clearly appears that at high temperature the core swollen by shell monomers is also covered by a shell highly enriched in pNNPAM. At intermediate temperatures $\left(35^{\circ} \mathrm{C}\right)$, the core starts to swell, but it is hindered by the interpenetrated network of shell monomers. As a result, the size of the interpenetrating core polymer (pNNPAM and pNIPMAM) increases somewhat but less than a pure pNIPMAM core, 'pushing' the pure shell monomers at its surface and thus forming a still insoluble pNNPAM shell around the core. It is thus the conjunction of the insoluble interpenetrated network of shell monomers, and the formation of a "true" shell, which seems to hinder the swelling and to generate the progressive linear swelling response found in our previous works. ${ }^{27,36}$

In previous work, we have used a combination of measurements on different particles under different contrast conditions, under the assumption of identical structures and sizes, in order to estimate the shell monomer profile ${ }^{46}$. We have measured the density profiles of pNIPMAM-pNNPAM core-shell microgels with $\mathrm{H}$-core and $\mathrm{H}$-shell, and of a H-core with a contrast matched D7-shell. From these measurements on different particles we calculated the estimated density profile of the D7-shell. The comparison with the unambiguous measurement provided here is given in Figure 6, clearly emphasizing the necessity of the present approach. 


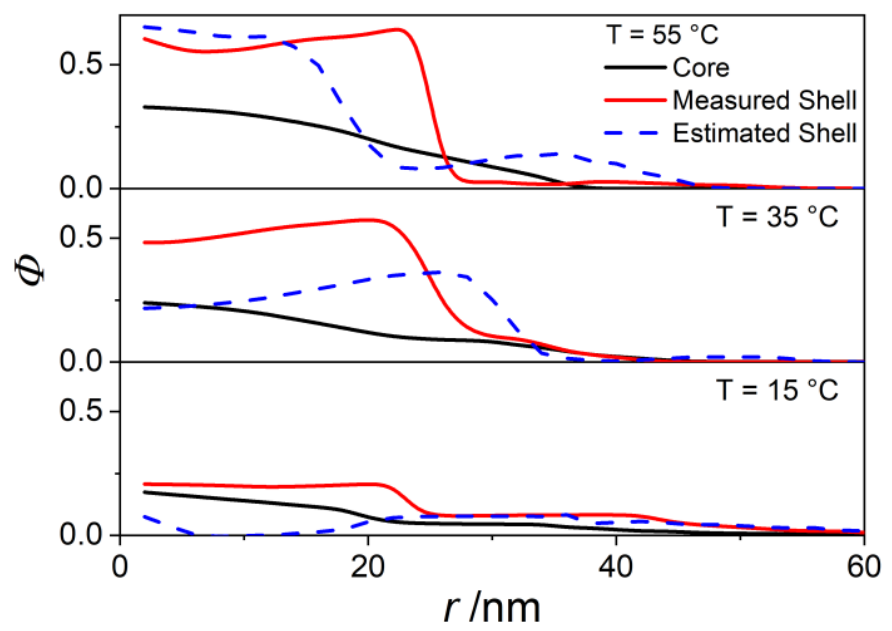

Figure 6: Core and shell density profiles of a H-pNIPMAM core with a D7-pNNPAM shell. The data of the "Estimated shell" are adapted from Cors et al. ${ }^{46}$ and the "Measured shell" profiles are the results from the present article.

The available detail on local monomer concentrations can also be used to forward a more molecular interpretation of linear swelling. Indeed, the ratio of the monomer densities plotted in the insets of Figure 5 illustrates the existence of zones of different composition. In case of statistical copolymer microgels the transition temperature was found to depend linearly on the comonomer composition. In analogy with such statistical copolymer microgels, ${ }^{58}$ one can assume an effective transition temperature given by some weighted average of the two polymers, leading to a gradient in transition temperatures. Quite counterintuitively, the swelling of the core induces a fuzzier interface and higher density of core monomers at the periphery of the core, which would thus swell first, followed at lower temperatures by the main part of the core. In the swollen state at $15{ }^{\circ} \mathrm{C}$, finally, the core and the shell monomers have low monomer densities across the entire particle (below $20 \%$ ) and approximately the same extent of ca. $55 \mathrm{~nm}$.

\section{Conclusion}

Our contrast-matching SANS experiments have provided direct access to the radial density profile of the shell monomers in core-shell microgels, proving a surprising non classical spatial distribution of the latter within the microgel particle. This has been suspected in previous work, ${ }^{46}$ where the profile was estimated using severe assumptions of identical synthesis in presence and absence of deuteration. The present approach on a single partially contrast-matched particle shows the limitations caused by these assumptions, and confirms the presence of a strongly interpenetrated network in the centre of the particle.

Our main result is thus that, instead of an external "corset" effect as previously assumed by Zeiser et al., ${ }^{27}$ the shell monomers build up both a small external shell and an internal skeleton with a density gradient (as 
demonstrated by the non constant ratios in the insets of Figure 5) which hinders the immediate swelling of the entire core. Indeed, a homogeneous mixture of core and shell monomers throughout the particle would lead to a single swelling step, whereas zones of different composition would respond at different temperatures. Although a mechanical model of the resulting microgel particle is out of reach for the present article, one may conjecture that this interpenetrated structure of varying local concentration causes the progressive linear swelling between the transition temperatures of the two monomers. Presumably the linear swelling response is thus related to a particular form of the monomer profiles. One may also suspect that the latter depends on the size of the core and possibly on the thickness of the shell layer. This would be the case, e.g., in presence of a finite penetration depth of the shell into the core. Then the degree of interpenetration would be controlled by the ratio of this depth to the core radius. In the future thus we aim at a better control of the interpenetration of the shell monomer into the core, either by varying synthesis parameters, or by exploring the effect of microgel size and shell thickness. The form-free multi-shell model combined with contrast-matching will be a valuable tool in this endeavour, as density profiles of all constituents are readily obtained. This approach might allow to engineer the properties of such core-shell microgels in a different way compared to the present synthesis. Moreover, it remains to be studied whether the observed behaviour is a peculiarity of these specific monomers, or if it is also occurring with other acrylamides when used in a two-step synthesis of core-shell particles.

\section{Acknowledgements}

The authors are thankful for support by the joint ANR/DFG CoreShellGel project, Grant ANR-14-CE35-0008-01 of the French Agence Nationale de la Recherche, and Grant HE2995/5-1 by Deutsche Forschungsgemeinschaft. This work is based on experiments performed at the KWS-1 instrument operated by JCNS at the Heinz Maier-Leibnitz Zentrum (MLZ, Garching, Germany) and PA20 at LLB (CEA, Saclay). We thank Ina Ehring (Bielefeld) for the help with the synthesis. 


\section{References}

(1) Murray, M.; Snowden, M. The preparation, characterisation and applications of colloidal microgels. Advances in Colloid and Interface Science 1995, 54, 73-91.

(2) Saunders, B. R.; Vincent, B. Microgel particles as model colloids: theory, properties and applications. Advances in Colloid and Interface Science 1999, 80, 1-25.

(3) Nayak, S.; Lyon, L. A. Soft Nanotechnology with Soft Nanoparticles. Angewandte Chemie International Edition 2005, 44, 7686-7708.

(4) Fernández-Barbero, A.; Suárez, I. J.; Sierra-Martn, B.; Fernández-Nieves, A.; de las Nieves, F. J.; Marquez, M.; Rubio-Retama, J.; López-Cabarcos, E. Gels and microgels for nanotechnological applications. Advances in Colloid and Interface Science 2009, 147-148, 88-108.

(5) Lapeyre, V.; Renaudie, N.; Dechezelles, J.-F.; Saadaoui, H.; Ravaine, S.; Ravaine, V. Multiresponsive Hybrid Microgels and Hollow Capsules with a Layered Structure. Langmuir 2009, 25, 4659-4667.

(6) Saunders, B. R.; Laajam, N.; Daly, E.; Teow, S.; Hu, X.; Stepto, R. Microgels: From responsive polymer colloids to biomaterials. Advances in Colloid and Interface Science 2009, 147-148, 251-262.

(7) Richtering, W.; Saunders, B. R. Gel architectures and their complexity. Soft Matter 2014, 10, 3695-3702.

(8) Destribats, M.; Eyharts, M.; Lapeyre, V.; Sellier, E.; Varga, I.; Ravaine, V.; Schmitt, V. Impact of pNIPAM Microgel Size on Its Ability To Stabilize Pickering Emulsions. Langmuir 2014, 30, 1768-1777.

(9) Plamper, F. A.; Richtering, W. Functional Microgels and Microgel Systems. Accounts of Chemical Research 2017, 50, 131-140.

(10) Karg, M.; Pich, A.; Hellweg, T.; Hoare, T.; Lyon, L. A.; Crassous, J. J.; Suzuki, D.; Gumerov, R. A.; Schneider, S.; Potemkin, I. I.; Richtering, W. Nanogels and Microgels: From Model Colloids to Applications, Recent Developments, and Future Trends. Langmuir 2019, 35, 6231-6255.

(11) Lu, Y.; Mei, Y.; Drechsler, M.; Ballauff, M. Thermosensitive CoreShell Particles as Carriers for Ag Nanoparticles: Modulating the Catalytic Activity by a Phase Transition in Networks. Angewandte Chemie International Edition 2006, 45, 813-816.

(12) Lu, Y.; Yuan, J.; Polzer, F.; Drechsler, M.; Preussner, J. In Situ Growth of Catalytic Active Au-Pt Bimetallic Nanorods in Thermoresponsive Core-Shell Microgels. ACS Nano 2010, 4, 7078-7086.

(13) Suzuki, D.; Kawaguchi, H. Gold Nanoparticle Localization at the Core Surface by Using Thermosensitive Core-Shell Particles as a Template. Langmuir 2005, 21, 12016-12024.

(14) Malmsten, M. Microgel Suspensions; Wiley-VCH Verlag GmbH \& Co. KGaA, 2011; pp 375-405.

(15) Labie, H.; Perro, A.; Lapeyre, V.; Goudeau, B.; Catargi, B.; Auzély, R.; Ravaine, V. Sealing hyaluronic acid microgels with oppositely-charged polypeptides: A simple strategy for packaging hydrophilic drugs with on-demand release. Journal of Colloid and Interface Science 2019, 535, 16-27.

(16) Li, X.; Gao, Y.; Serpe, M. Stimuli-Responsive Assemblies for Sensing Applications. Gels 2016, $2,8$.

(17) Zhou, X.; Nie, J.; Du, B. Functionalized Ionic Microgel Sensor Array for Colorimetric Detection and Discrimination of Metal Ions. ACS Applied Materials \& Interfaces 2017, 9, 20913-20921. 
(18) Islam, M. R.; Azimi, S.; Teimoory, F.; Loppnow, G.; Serpe, M. J. Isolation of RNA from a mixture and its detection by utilizing a microgel-based optical device. Canadian Journal of Chemistry 2018, 96, 1079-1086.

(19) Shu, T.; Shen, Q.; Wan, Y.; Zhang, W.; Su, L.; Zhang, X.; Serpe, M. J. Silver nanoparticle-loaded microgelbased etalons for $\mathrm{H}_{2} \mathrm{O}_{2}$ sensing. RSC Advances 2018, 8, 15567-15574.

(20) Islam, M. R.; Irvine, J.; Serpe, M. J. Photothermally Induced Optical Property Changes of Poly $(N$ isopropylacrylamide) Microgel-Based Etalons. ACS Applied Materials \& Interfaces 2015, 7, 24370-24376.

(21) Wei, M.; Serpe, M. J. Temperature-Light Dual-Responsive Au@PNIPAm Core-Shell Microgel-Based Optical Devices. Particle \& Particle Systems Characterization 2018, 36, 1800326.

(22) Burmistrova, A.; von Klitzing, R. Control of number density and swelling/shrinking behavior of P(NIPAMAAc) particles at solid surfaces. Journal of Materials Chemistry 2010, $20,3502$.

(23) Li, X.; Cai, X.; Gao, Y.; Serpe, M. J. Reversible bidirectional bending of hydrogel-based bilayer actuators. Journal of Materials Chemistry B 2017, 5, 2804-2812.

(24) Uhlig, K.; Wegener, T.; Hertle, Y.; Bookhold, J.; Jaeger, M.; Hellweg, T.; Fery, A.; Duschl, C. Thermoresponsive Microgel Coatings as Versatile Functional Compounds for Novel Cell Manipulation Tools. Polymers 2018, 10, 656 .

(25) Iwai, K.; Matsumura, Y.; Uchiyama, S.; de Silva, A.P. Development of fluorescent microgel thermometers based on thermo-responsive polymers and their modulation of sensitivity range. Journal of Materials Chemistry $\mathbf{2 0 0 5}$, $15,2796$.

(26) Keerl, M.; Smirnovas, V.; Winter, R.; Richtering, W. Copolymer Microgels from Mono- and Disubstituted Acrylamides: Phase Behavior and Hydrogen Bonds. Macromolecules 2008, 41, 6830-6836.

(27) Zeiser, M.; Freudensprung, I.; Hellweg, T. Linearly thermoresponsive coreshell microgels: Towards a new class of nanoactuators. Polymer 2012, 53, 6096-6101.

(28) Cors, M.; Wiehemeier, L.; Oberdisse, J.; Hellweg, T. Deuteration-Induced Volume Phase Transition Temperature Shift of PNIPMAM Microgels. Polymers 2019, 11, 620.

(29) Monte, G. D.; Ninarello, A.; Camerin, F.; Rovigatti, L.; Gnan, N.; Zaccarelli, E. Numerical insights on ionic microgels: structure and swelling behaviour. Soft Matter 2019, 15, 8113-8128.

(30) Gelissen, A. P. H.; Scotti, A.; Turnhoff, S. K.; Janssen, C.; Radulescu, A.; Pich, A.; Rudov, A. A.; Potemkin, I. I.; Richtering, W. An anionic shell shields a cationic core allowing for uptake and release of polyelectrolytes within coreshell responsive microgels. Soft Matter 2018, 14, 4287-4299.

(31) Hellweg, T. Responsive core-shell microgels: Synthesis, characterization, and possible applications. Journal of Polymer Science Part B: Polymer Physics 2013, 51, 1073-1083.

(32) Dubbert, J.; Nothdurft, K.; Karg, M.; Richtering, W. Core-Shell-Shell and Hollow Double-Shell Microgels with Advanced Temperature Responsiveness. Macromolecular Rapid Communications 2014, 36, 159-164.

(33) Brugnoni, M.; Scotti, A.; Rudov, A. A.; Gelissen, A. P. H.; Caumanns, T.; Radulescu, A.; Eckert, T.; Pich, A.; Potemkin, I. I.; Richtering, W. Swelling of a Responsive Network within Different Constraints in Multi-Thermosensitive Microgels. Macromolecules 2018, 51, 2662-2671.

(34) Dubbert, J.; Honold, T.; Pedersen, J. S.; Radulescu, A.; Drechsler, M.; Karg, M.; Richtering, W. How Hollow Are Thermoresponsive Hollow Nanogels? Macromolecules 2014, 47, 8700-8708. 
(35) Schmid, A. J.; Dubbert, J.; Rudov, A. A.; Pedersen, J. S.; Lindner, P.; Karg, M.; Potemkin, I. I.; Richtering, W. Multi-Shell Hollow Nanogels with Responsive Shell Permeability. Scientific Reports 2016, 6.

(36) Cors, M.; Wrede, O.; Genix, A.-C.; Anselmetti, D.; Oberdisse, J.; Hellweg, T. CoreShell Microgel-Based Surface Coatings with Linear Thermoresponse. Langmuir 2017, 33, 6804-6811.

(37) Wiehemeier, L.; Cors, M.; Wrede, O.; Oberdisse, J.; Hellweg, T.; Kottke, T. Swelling behaviour of coreshell microgels in $\mathrm{H}_{2} \mathrm{O}$, analysed by temperature-dependent FTIR spectroscopy. Physical Chemistry Chemical Physics 2019, $21,572-580$.

(38) Berndt, I.; Richtering, W. Doubly temperature sensitive core-shell microgels. Macromolecules 2003, 36, 87808785.

(39) Berndt, I.; Pedersen, J. S.; Richtering, W. Structure of Multiresponsive Intelligent Core-Shell Microgels. Journal of the American Chemical Society 2005, 127, 9372-9373.

(40) Berndt, I.; Pedersen, J. S.; Lindner, P.; Richtering, W. Influence of Shell Thickness and Cross-Link Density on the Structure of Temperature-Sensitive Poly- $N$-Isopropylacrylamide-Poly- $N$-Isopropylmethacrylamide Core-Shell Microgels Investigated by Small-Angle Neutron Scattering. Langmuir 2006, 22, 459-468.

(41) Berndt, I.; Pedersen, J. S.; Richtering, W. Temperature-Sensitive CoreShell Microgel Particles with Dense Shell. Angewandte Chemie International Edition 2006, 45, 1737-1741.

(42) Otto, P.; Bergmann, S.; Sandmeyer, A.; Dirksen, M.; Wrede, O.; Hellweg, T.; Huser, T. Resolving the internal morphology of coreshell microgels with super-resolution fluorescence microscopy. Nanoscale Advances 2020,

(43) Gelissen, A. P. H.; Oppermann, A.; Caumanns, T.; Hebbeker, P.; Turnhoff, S. K.; Tiwari, R.; Eisold, S.; Simon, U.; Lu, Y.; Mayer, J.; Richtering, W.; Walther, A.; Wöll, D. 3D Structures of Responsive Nanocompartmentalized Microgels. Nano Letters 2016, 16, 7295-7301.

(44) Berndt, I.; Popescu, C.; Wortmann, F.-J.; Richtering, W. Mechanics versus Thermodynamics: Swelling in Multiple-Temperature-Sensitive CoreShell Microgels. Angewandte Chemie International Edition 2006, 45, 1081-1085.

(45) Virtanen, O. L. J.; Mourran, A.; Pinard, P. T.; Richtering, W. Persulfate initiated ultra-low cross-linked poly( $N$ isopropylacrylamide) microgels possess an unusual inverted cross-linking structure. Soft Matter 2016, 12, 3919-3928.

(46) Cors, M.; Wrede, O.; Wiehemeier, L.; Feoktystov, A.; Cousin, F.; Hellweg, T.; Oberdisse, J. Spatial distribution of core monomers in acrylamide-based core-shell microgels with linear swelling behaviour. Scientific Reports 2019, 9 , 13812.

(47) McGreevy, R. L.; Pusztai, L. Reverse Monte Carlo Simulation: A New Technique for the Determination of Disordered Structures. Molecular Simulation 1988, 1, 359-367.

(48) Pethes, I.; Pusztai, L. Reverse Monte Carlo modeling of liquid water with the explicit use of the SPC/E interatomic potential. The Journal of Chemical Physics 2017, 146, 064506.

(49) Oberdisse, J.; Hine, P.; Pyckhout-Hintzen, W. Structure of interacting aggregates of silicananoparticles in a polymer matrix: small-angle scattering and reverse Monte Carlo simulations. Soft Matter 2007, 3, 476-485.

(50) Cors, M.; Wiehemeier, L.; Hertle, Y.; Feoktystov, A.; Cousin, F.; Hellweg, T.; Oberdisse, J. Determination of Internal Density Profiles of Smart Acrylamide-Based Microgels by Small-Angle Neutron Scattering: A Multishell Reverse Monte Carlo Approach. Langmuir 2018, 34, 15403-15415. 
(51) Oliveira, C. L. P.; Gerbelli, B. B.; Silva, E. R. T.; Nallet, F.; Navailles, L.; Oliveira, E. A.; Pedersen, J. S. Gaussian deconvolution: a useful method for a form-free modeling of scattering data from mono- and multilayered planar systems. Journal of Applied Crystallography 2012, 45, 1278-1286.

(52) Hirano, T.; Nakamura, K.; Kamikubo, T.; Ishii, S.; Tani, K.; Mori, T.; Sato, T. Hydrogen-bond-assisted syndiotactic-specific radical polymerizations of $N$-alkylacrylamides: The effect of the $N$-substituents on the stereospecificities and unusual large hysteresis in the phase-transition behavior of aqueous solution of syndiotactic poly( $N$-n-propylacrylamide). Journal of Polymer Science Part A: Polymer Chemistry 2008, 46, 4575-4583.

(53) Chaboussant, G.; Désert, S.; Lavie, P.; Brûlet, A. PA20 : A new SANS and GISANS project for soft matter, materials and magnetism. Journal of Physics: Conference Series 2012, 340, 012002.

(54) Feoktystov, A. V.; Frielinghaus, H.; Di, Z.; Jaksch, S.; Pipich, V.; Appavou, M.-S.; Babcock, E.; Hanslik, R.; Engels, R.; Kemmerling, G.; Kleines, H.; Ioffe, A.; Richter, D.; Brückel, T. KWS-1 high-resolution small-angle neutron scattering instrument at JCNS: current state. Journal of Applied Crystallography 2015, 48, 61-70.

(55) Debord, S. B.; Lyon, L. A. Influence of Particle Volume Fraction on Packing in Responsive Hydrogel Colloidal Crystals. The Journal of Physical Chemistry B 2003, 107, 2927-2932.

(56) Hammouda, B. Small-Angle Scattering From Branched Polymers. Macromolecular Theory and Simulations 2012, 21, 372-381.

(57) Balaceanu, A.; Demco, D. E.; Möller, M.; Pich, A. Heterogeneous Morphology of Random Copolymer Microgels as Reflected in Temperature-Induced Volume Transition and 1H High-Resolution Transverse Relaxation NMR. Macromolecular Chemistry and Physics 2011, 212, 2467-2477.

(58) Wedel, B.; Zeiser, M.; Hellweg, T. Non NIPAM Based Smart Microgels: Systematic Variation of the Volume Phase Transition Temperature by Copolymerization. Zeitschrift für Physikalische Chemie 2012, 226, 737-748.

(59) Wu, Y.; Wiese, S.; Balaceanu, A.; Richtering, W.; Pich, A. Behavior of Temperature-Responsive Copolymer Microgels at the Oil/Water Interface. Langmuir 2014, 30, 7660-7669.

(60) Crassous, J. J.; Mihut, A. M.; Månsson, L. K.; Schurtenberger, P. Anisotropic responsive microgels with tuneable shape and interactions. Nanoscale 2015, 7, 15971-15982.

(61) von Nessen, K.; Karg, M.; Hellweg, T. Thermoresponsive poly-( $N$-isopropylmethacrylamide) microgels: Tailoring particle size by interfacial tension control. Polymer 2013, 54, 5499-5510. 